\title{
Simulation study of GaAsP/Si tandem cells including the impact of threading dislocations on the luminescent coupling between the cells
}

Arthur Onno, Nils-Peter Harder, Lars Oberbeck, Huiyun Liu

Arthur Onno, Nils-Peter Harder, Lars Oberbeck, Huiyun Liu, "Simulation study of GaAsP/Si tandem cells including the impact of threading dislocations on the luminescent coupling between the cells," Proc. SPIE 9743, Physics, Simulation, and Photonic Engineering of Photovoltaic Devices V, 97431B (14 March 2016); doi: 10.1117/12.2211113

SPIE. Event: SPIE OPTO, 2016, San Francisco, California, United States 


\title{
Simulation study of GaAsP/Si tandem cells including the impact of threading dislocations on the luminescent coupling between the cells
}

\author{
Arthur Onno*a , Nils-Peter Harder ${ }^{\mathrm{b}}$, Lars Oberbeck ${ }^{\mathrm{b}}$, Huiyun Liu ${ }^{\mathrm{a}}$ \\ ${ }^{a}$ Department of Electronic and Electrical Engineering, UCL, London WC1E 7JE, United Kingdom \\ ${ }^{\mathrm{b}}$ TOTAL Marketing Services, New Energies Division, 92069 Paris La Défense Cedex, France
}

\begin{abstract}
A model, derived from the detailed balance model from Shockley and Queisser, has been adapted to monolithically grown GaAsP/Si tandem dual junction solar cells. In this architecture, due to the difference of lattice parameters between the silicon bottom cell - acting as the substrate - and the GaAsP top cell, threading dislocations (TDs) arise at the III$\mathrm{V} / \mathrm{Si}$ interface and propagate in the top cell. These TDs act as non-radiative recombination centers, degrading the performances of the tandem cell. Our model takes into account the impact of TDs by integrating the NTT model developed by Yamaguchi et. al.. Two surface geometries have been investigated: flat and ideally textured. Finally the model considers the luminescent coupling (LC) between the cells due to reemitted photons from the top cell cascading to the bottom cell.

Without dislocations, LC allows a greater flexibility in the cell design by rebalancing the currents between the two cells when the top cell presents a higher short-circuit current. However we show that, as the TD density (TDD) increases, nonradiative recombinations take over radiative recombinations in the top cell and the LC is quenched. As a result, nonoptimized tandem cells with higher short-circuit current in the top cell experience a very fast degradation of efficiency for TDDs over $10^{4} \mathrm{~cm}^{-2}$. On the other hand optimized cells with matching currents only experience a small efficiency drop for TDDs up to $10^{5} \mathrm{~cm}^{-2}$. High TDD cells therefore need to be current-matched for optimal performances as the flexibility due to LC is lost.
\end{abstract}

Keywords: Dual-junction, Silicon, GaAsP, Threading dislocation density, Luminescent coupling, Model

\section{INTRODUCTION}

With 25.6\% record efficiency [1] and 25.0\% achieved utilizing industry-ready equipment [2], low-cost crystalline silicon (c-Si) photovoltaic technology, dominant on the market, is already very close to its theoretical maximum of approximately $29 \%[3,4]$ with a limited margin of improvement using standard technologies. Although achieving efficiencies close to $40 \%$ without concentration [5-6], III-V-based multijunction solar cells are extremely expensive, making them impractical for large-scale deployments without high concentration systems.

By combining the large-scale optimized supply chain of the industrial silicon platform with the high efficiency of III-Vbased multijunction technology, epitaxially grown III-V on Si multijunction solar cells present an elegant pathway to overcome the physical limitations of the c-Si technology while keeping the advantage of a relatively low-cost substrate. Good progress has been achieved recently using a GaAsP/Si dual-junction architecture where a $1.7 \mathrm{eV}$ GaAsP top cell is epitaxially grown by Molecular Beam Epitaxy (MBE) or Metal-Organic Chemical Vapor Deposition (MOCVD) on a Si bottom cell acting as a substrate [7-9]. The main challenge resides in the difference of lattice-parameter between $\mathrm{Si}$ and $1.7 \mathrm{eV} \mathrm{GaAsP}$ which causes a build-up of strain in the III-V epilayers, leading to the formation of Threading Dislocations (TDs). These TDs act as recombination centers, reducing the diffusion length and lifetime of the minority carriers and strongly impeding the performances of the top cell. As a result the efficiency of the current-matched GaAsP/Si dualjunction is reduced.

TDs also strongly impact the Luminescent Coupling (LC) between the cells. LC comes from photons reemitted from radiative recombinations in the top cell cascading to the bottom cell where they are strongly absorbed and participate in the photocurrent. This phenomenon can greatly improve the performances of tandem cells with a non-optimized top cell - where a lower than optimal bandgap or a higher than optimal thickness causes the top cell photocurrent to be higher

Physics, Simulation, and Photonic Engineering of Photovoltaic Devices V, edited by Alexandre Freundlich, Laurent Lombez, Masakazu Sugiyama, Proc. of SPIE Vol. 9743, 97431B · ( 2016 SPIE CCC code: $0277-786 \mathrm{X} / 16 / \$ 18 \cdot$ doi: $10.1117 / 12.2211113$ 
than ideal - by rebalancing the curents in the two cells [10]. In the case of a high TD Density (TDD), non-radiative recombinations take over radiative ones in the top cell and the LC is quenched.

In order to investigate the impact of the TDD and LC on the performances of GaAsP/Si tandem dual-junction solar cells, we have developed an inclusive yet simple model based on the Shockley-Queisser detailed balance model [3]. The model takes into account the composition and the resulting bandgap of the $\mathrm{GaAs}_{\mathrm{x}} \mathrm{P}_{1-\mathrm{x}}$ top cell, its thickness and two absorption models: flat and ideally textured Lambertian top surface.

\section{METHOD}

The architecture investigated here is presented in Figure 1. The dual-junction GaAsP/Si cell is a two-terminal seriesconnected tandem structure with current-matched cells. The cells are connected through a high bandgap window buffer similar to the metamorphic GaAsP buffer on a GaP nucleation layer presented in Refs. [7-9]. Due to its high bandgap, absorption is neglected in this buffer. The top cell composition varies in the direct bandgap domain of $\mathrm{GaAs}_{\mathrm{x}} \mathrm{P}_{1-\mathrm{x}}$, with the As content varying from $x=0.55\left(E_{g}=1.98 \mathrm{eV}\right)$ to $1\left(E_{\mathrm{g}}=1.42 \mathrm{eV}\right)$. Its thickness varies from $0.2 \mu \mathrm{m}$ to $5 \mu \mathrm{m}$ with a fixed emitter thickness of $0.1 \mu \mathrm{m}$. Because of its relatively high direct bandgap, Auger recombinations are neglected in the top cell and TD-related Shockley-Read-Hall (SRH) recombinations are the only source of bulk non-radiative recombinations considered there. The Si bottom cell thickness is fixed at $150 \mu \mathrm{m}$, representative of the c-Si industry standard. We consider very high quality $\mathrm{Si}$ with a negligible defect density. Thus we neglect SRH recombinations in the bottom cell and only take Auger recombinations into account for bulk non-radiative recombinations. Surface recombinations are ignored as we concentrate on the impact of the TDD and LC on the theoretical efficiency of the GaAsP/Si architecture.

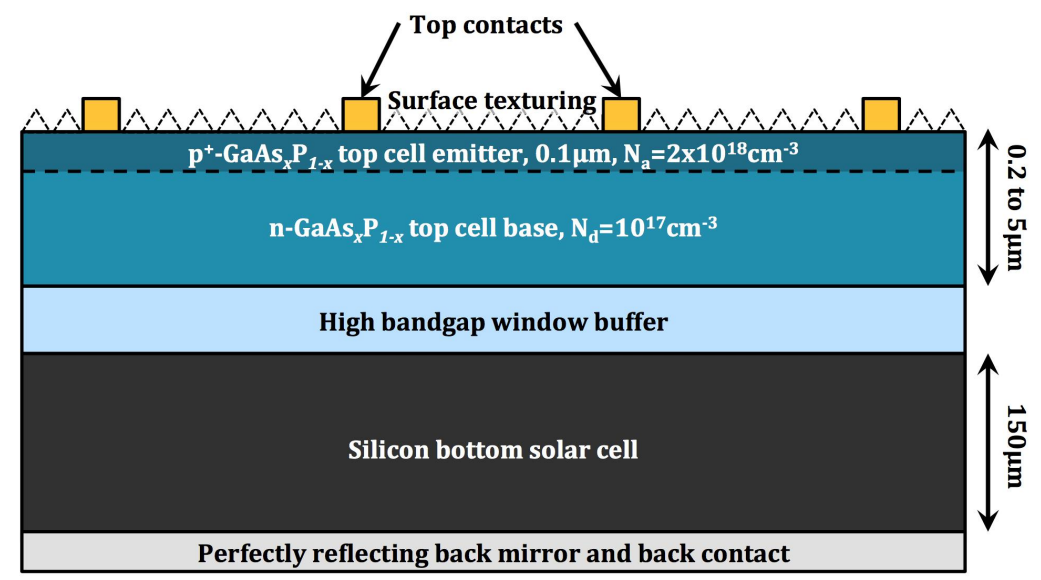

Figure 1. Detail of the GaAsP/Si dual junction architecture investigated.

We consider a perfectly reflecting back-mirror on the Si bottom surface. Two absorption models, corresponding to two surface geometries, are taken into account. The first absorption model corresponds to the simple case of a flat surface resulting in a one pass through the top cell and a two pass through the bottom cell as shown in Equations (1-2).

$$
\begin{gathered}
a_{\text {front }, \text { top }}(\lambda)=1-e^{-\alpha_{G a A s P}(\lambda) L_{t o p}} \\
a_{\text {front }, \text { bot }}(\lambda)=1-e^{-2 \alpha_{S i}(\lambda) L_{b o t}}
\end{gathered}
$$

where $a_{\text {front,top/bot }}(\lambda)$ is the wavelength-dependent front absorptivity of the top/bottom cells, $\alpha_{\text {GaAsP/Si }}(\lambda)$ is the wavelengthdependent absorption coefficient of GaAsP/Si and $L_{\text {top/bot }}$ is the thickness of the top/bottom cell. The second model, detailed in Equations (3-4), corresponds to a Lambertian top surface scattering the incoming light. For the bottom cell, as the top cell and buffer are windows for the wavelengths in the weakly absorbing limit, perfect light trapping is obtained [11]. 


$$
\begin{gathered}
a_{\text {front }, \text { top }}(\lambda)=2 \int_{0}^{\frac{\pi}{2}}\left(1-e^{-\alpha_{G a A s P}(\lambda) \frac{L_{t o p}}{\cos \theta}}\right) \cos \theta \sin \theta d \theta \\
a_{\text {front }, \text { bot }}(\lambda)=\frac{4 n_{\text {ref }}^{2} \alpha_{S i}(\lambda) L_{\text {bot }}}{1+4 n_{\text {ref }}^{2} \alpha_{S i}(\lambda) L_{\text {bot }}}
\end{gathered}
$$

where $n_{r e f}=3.5$ is the refractive index of Si. As the top cell does not have a back-mirror, top cell back-surface absorption for both models also needs to be considered for the blackbody thermal equilibrium, as shown in Equation (5) (flat surface) and (6) (Lambertian surface).

$$
\begin{aligned}
a_{\text {back }, \text { top }}(\lambda)=2 n_{\text {ref }}^{2}\left(\int_{0}^{\theta_{e s c}}\left(1-e^{-\alpha_{G a A s P}(\lambda) \frac{L_{\text {top }}}{\cos \theta}}\right) \cos \theta \sin \theta d \theta+\int_{\theta_{\text {esc }}}^{\frac{\pi}{2}}\left(1-e^{-2 \alpha_{G a A s P}(\lambda) \frac{L_{\text {top }}}{\cos \theta}}\right) \cos \theta \sin \theta d \theta\right) \\
a_{\text {back,top }}(\lambda)=2 n_{\text {ref }}^{2}\left(\frac { 2 \theta _ { \text { esc } } } { \pi } \int _ { 0 } ^ { \frac { \pi } { 2 } } \left(1-e^{\left.-\alpha_{G a A s P}(\lambda) \frac{L_{t o p}}{\cos \theta}\right) \cos \theta \sin \theta d \theta}\right.\right. \\
\left.+\left(1-\frac{2 \theta_{e s c}}{\pi}\right) \int_{0}^{\frac{\pi}{2}} \int_{0}^{\frac{\pi}{2}}\left(1-e^{-2 \alpha_{G a A s P}(\lambda)\left(\frac{L_{t o p}}{\cos \theta}+\frac{L_{t o p}}{\cos \theta^{\prime}}\right)}\right) \cos \theta \cos \theta^{\prime} \sin \theta d \theta d \theta^{\prime}\right)
\end{aligned}
$$

where $a_{\text {back,top }}$ is the absorptivity of the top cell from its back surface and $\theta_{\text {esc }}$ is the escape angle.

For each cell we consider the well-known general diode equation:

$$
J(V)=J_{s c}+q R_{r, \text { rad }}\left(1-e^{\frac{q V}{k_{B} T}}\right)+q \sum_{m} R_{r, m}\left(1-e^{\frac{q V}{n_{m} k_{B} T}}\right)
$$

where $J$ is the current density, $V$ the voltage, $J_{s c}$ the short-circuit current density, $q$ the elementary charge, $R_{r, r a d}$ the radiative recombination rate, $k_{B}$ the Boltzmann constant, $T$ the temperature fixed at $300 \mathrm{~K}, R_{r, m}$ the non-radiative recombination rate associated with the non-radiative recombination process $m$ and $n_{m}$ the corresponding ideality factor.

As the only sources of non-radiative recombinations considered are the SRH recombinations in the GaAsP top cell and the Auger recombinations in the bottom cell we have:

$$
\begin{gathered}
J_{\text {top }}\left(V_{\text {top }}\right)=J_{s c, \text { top }}+q R_{r, \text { rad }, \text { top }}\left(1-e^{\frac{q V_{\text {top }}}{k_{B} T}}\right)+R_{r, S R H}\left(1-e^{\frac{q V_{\text {top }}}{2 k_{B} T}}\right) \\
J_{\text {bot }}\left(V_{\text {bot }}\right)=J_{s c, \text { bot }}+q R_{r, \text { rad }, \text { bot }}\left(1-e^{\frac{q V_{b o t}}{k_{B} T}}\right)+R_{r, \text { Auger }}\left(1-e^{\frac{3 q V_{b o t}}{2 k_{B} T}}\right)
\end{gathered}
$$

where $R_{r, S R H}$ is the short-circuit TD-related SRH recombination rate in the top cell with the associated ideality factor of 2 and $R_{r, \text { Auger }}$ is the short-circuit Auger recombination rate in the Si bottom cell with the associated ideality factor of 2/3.

The short-circuit current density $J_{s c}$, equal to the photocurrent in the hypothesis of no recombinations at $V=0$, is given in the top and bottom cells by:

$$
\begin{gathered}
J_{s c, \text { top }}=q \int_{0}^{+\infty}\left(\frac{\lambda}{h c}\right) I(\lambda) a_{\text {front }, \text { top }}(\lambda) d \lambda \\
J_{s c, b o t}=q \int_{0}^{+\infty}\left(\frac{\lambda}{h c}\right) I(\lambda)\left(1-a_{\text {front }, \text { top }}(\lambda)\right) a_{\text {front }, \text { bot }}(\lambda) d \lambda
\end{gathered}
$$

where $\lambda$ is the incoming photon wavelength, $h$ is the Planck constant, $c$ is the speed of light and $I(\lambda)$ is the wavelengthdependent AM1.5 irradiance from Ref [12].

Considering the Bose-Einstein approximation $\left(\mathrm{E}>>k_{B} T\right)$ for both cells, the radiative recombination rate is given by the classic formula [13]: 


$$
R_{r, \text { rad }}=\int_{0}^{+\infty} \frac{2 \pi a(E) E^{2}}{h^{3} c^{2}} e^{-\frac{E}{k_{B} T}}=\int_{0}^{+\infty} \frac{2 \pi c a(\lambda)}{\lambda^{4}} e^{-\frac{h c}{\lambda k_{B} T}} d \lambda
$$

where $a(\lambda)$ is the absorptivity of the cell through both its top and bottom surfaces. In the case of the Si bottom cell $a_{b o t}=a_{\text {front bot }}$ as the back absorptivity is null because of the back-mirror. However, in the case of the top cell $a_{\text {top }}=a_{\text {front,top }}+a_{\text {back,top }}$.

Considering a low or intrinsic doping density in the Si bottom cell, the short-circuit Auger recombination rate $R_{r, \text { Auger }}$ is given by [4]:

$$
R_{r, \text { Auger }}=C_{\text {Auger }} L_{\text {bot }} n_{i, S i}^{3}
$$

where $C_{\text {Auger }}$ is the ambipolar Auger coefficient of $\mathrm{Si}$ and $n_{i, S i}$ is the intrinsic carrier concentration of Si, both from Ref. [14].

Using the NTT model developed by Yamaguchi et. al. [15], the diffusion length of minority carriers $L_{T D}$ associated with a TDD $\rho_{T D}$ is given by:

$$
L_{T D}=\sqrt{\frac{4}{\pi^{3} \rho_{T D}}}
$$

The short-circuit SRH recombination rate $R_{r, S R H}$, strongly dominated by recombinations in the depletion zone where the electron and hole densities are of the same order of magnitude, is therefore [15]:

$$
R_{r, S R H}=\frac{n_{i, G a A s P} W_{D}}{2} \frac{D_{p}}{L_{T D}^{2}}=\frac{\pi^{3} D_{p} \rho_{T D} n_{i, G a A s P} W_{D}}{8}
$$

where $n_{i, G a A s P}$ is the GaAsP intrinsic carrier concentration, $W_{D}$ the depletion zone width and $D_{p}$ the minority-carrier diffusion coefficient of holes as we consider a $\mathrm{p}^{+} / \mathrm{n}$ junction. The intrinsic carrier concentration of GaAsP is calculated using the classic relation with the bandgap and effective conduction/valence band densities of states. Similarly, the depletion width is obtained from the classic relation between the depletion width, the doping densities, the intrinsic carrier concentration and the relative permittivity. All the material-related parameters for GaAsP are extrapolated from Ref. [16].

For high TDD values, the collection efficiency of the top cell will also be impacted as the diffusion length of minority carriers becomes smaller than the distance the carriers have to travel to be extracted. This phenomenon will not impact the collection efficiency in the depletion zone and the emitter, even for high TDD values, as these regions are pretty thin. However, in an approximation of uniform generation rate, when the minority carriers diffusion length $L_{T D}$ becomes smaller than the thickness of the base $W_{\text {base }}$, the actual thickness of the top cell $L_{\text {top }}=W_{\text {base }}+W_{D}+W_{\text {emitter }}$, where $W_{\text {emitter }}$ is the thickness of the emitter, needs to be replaced by an effective thickness $L_{t o p}{ }^{\prime}=L_{T D}+W_{D}+W_{\text {emitter }}$ in the $J_{s c}$ calculations in Equations (10-11).

An additional process to take into account is LC between the cells. In the first approximation, considering the escape of reemitted photons from the cell as isotropic, the probability of a photon to escape through the top surface is $\theta_{\text {esc }} / \pi$ and the probability of cascading to the bottom cell is $1-\left(\theta_{\text {esc }} / \pi\right)$. As the reemitted photons have an energy close to the top cell bandgap, they are strongly absorbed in the bottom cell. All these cascading reemitted photons are therefore contributing to the bottom short-circuit current density. The current density boost from LC $J_{L C}$ is therefore given by:

$$
J_{L C}\left(V_{\text {top }}\right)=q\left(1-\frac{\theta_{\text {esc }}}{\pi}\right) R_{r, \text { rad }, \text { top }} e^{\frac{q V_{\text {top }}}{k_{B} T}}
$$

As the two cells are series-connected, the efficiency of the tandem junction solar cell is obtained by maximizing $J \times\left(V_{t o p}+V_{b o t}\right)$ under the condition $J_{t o p}=J_{b o t}$ :

$$
\begin{gathered}
J_{s c, \text { top }}+q R_{r, \text { rad }, \text { top }}\left(1-e^{\frac{q V_{t o p}}{k_{B} T}}\right)+R_{r, S R H}\left(1-e^{\frac{q V_{t o p}}{2 k_{B} T}}\right) \\
=J_{s c, b o t}+q\left(1-\frac{\theta_{e s c}}{\pi}\right) R_{r, \text { rad }, \text { top }} e^{\frac{q V_{t o p}}{k_{B} T}}+q R_{r, \text { rad }, \text { bot }}\left(1-e^{\frac{q V_{b o t}}{k_{B} T}}\right)+R_{r, \text { Auger }}\left(1-e^{\frac{3 q V_{b o t}}{2 k_{B} T}}\right)
\end{gathered}
$$




\section{RESULTS AND DISCUSSION}

Figure 2 presents the impact of the LC on the maximal theoretical efficiency of a Lambertian surface tandem GaAsP/Si dual junction solar cell. Both graphs are isoefficiency contours as a function of the bandgap and the thickness of the GaAsP top cell, with (a) and without (b) taking into account the impact of the LC. The dashed line represents the optimal bandgap-thickness combinations. The diamond-shaped dot is the particular case highlighted in Figure 3.
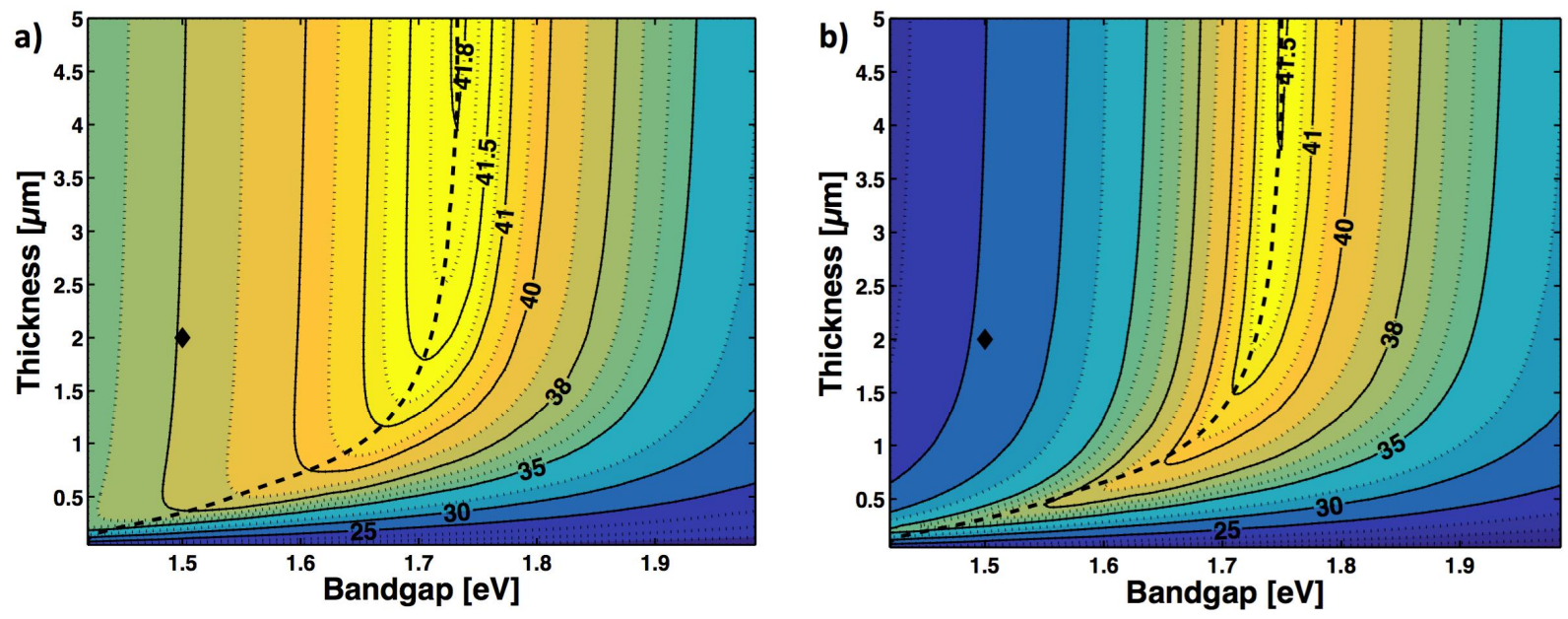

Figure 2. Theoretical maximum isoefficiency contours of a Lambertian surface $\mathrm{GaAsP} / \mathrm{Si}$ dual junction solar cell as a function of the top cell bandgap and thickness with (a) and without (b) taking into account the impact of the LC between the cells. The dashed line represents the optimal bandgap-thickness combinations. The diamond-shaped dot is the particular case exposed in Figure 3.

The impact of LC for non-optimized top cell bandgap-thickness combinations is apparent when a too high photocurrent is generated in the top cell, as it is the case on top and on the left of the dashed line with a too low bandgap/too high thickness for the top cell. The LC rebalances the currents between the top and bottom cells as shown in Figure 3, where the boost to the bottom cell $J_{s c}$ amounts to nearly $7 \mathrm{~mA} \cdot \mathrm{cm}^{-2}$ in the case of a $1.5 \mathrm{eV}, 2 \mu \mathrm{m}$-thick top cell. In that case the efficiency of the tandem solar cell is therefore not strongly impacted by an unoptimized structure thanks to the LC. However, when the bottom cell is the one producing a too high current (right of and under the dashed line), this cascading process cannot happen, as the photons reemitted from the bottom cell are too low energy to be absorbed in the top one.

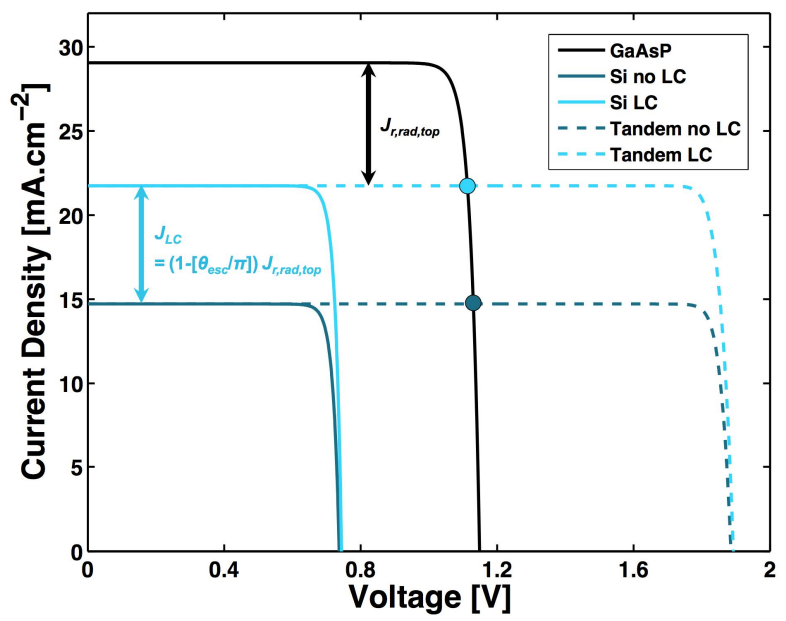

Figure 3. J-V characteristic of the GaAsP top cell (black), Si bottom cell (dark/light blue full line) and full dual junction cell (dark/light blue dashed line) in a tandem GaAsP/Si architecture with (light blue) and without (dark blue) taking into account LC. The top cell has a bandgap of $1.5 \mathrm{eV}$ and is $2 \mu \mathrm{m}$-thick. The light and dark blue dots represent the approximate maximal power point of the GaAsP top cell in each case. 
However, when a substantial level of TDs is accounted for, non-radiative SRH recombinations take over radiative recombinations and the LC is quenched. This is apparent on Figure 4a, which displays the boost $J_{L C}\left(V_{m p p}\right)$ to the bottom cell current density at maximum power point when taking into account LC. The iso-current density contours are calculated from Equation (16) as a function of the bandgap and the TDD of the top cell. As expected for low TDD values the boost due to $\mathrm{LC}$ is very dependent on the top cell bandgap. For bandgaps lower than the optimum of about $1.7 \mathrm{eV}$, the LC boost decreases steadily with the increase of the top cell bandgap. For bandgaps above the optimum, the LC boost is very small and does not strongly vary, indicative of the top cell being the limiting one in term of current density. On the contrary, for TDD values higher than $10^{5} \mathrm{~cm}^{-2}$, the current density boost due to LC decreases very fast with increasing TDD and its dependency with the top cell bandgap is reduced. As shown on Figure 4b, that displays the LC boost as a function of the TDD in the particular case of a $1.5 \mathrm{eV}$ top cell bandgap, the LC boost to the bottom cell current density decreases extremely fast with increasing TDD over $10^{4} \mathrm{~cm}^{-2}$, becoming negligible over $10^{6} \mathrm{~cm}^{-2}$.
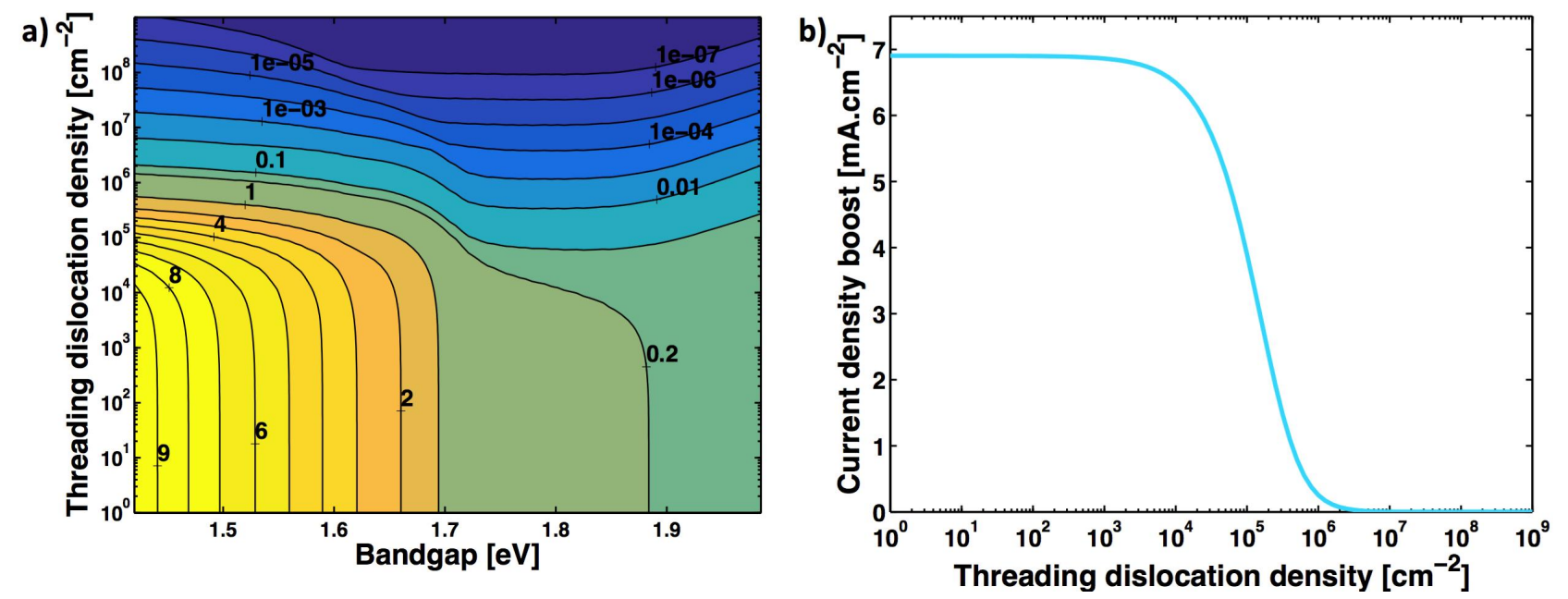

Figure 4. (a) Increase of the bottom cell current density (in $\mathrm{mA} . \mathrm{cm}^{-2}$ ) at maximum power point when taking into account LC. Iso-current density contours are given as a function of the top cell bandgap and TDD from Equation (16). Here a $2 \mu \mathrm{m}-$ thick top cell with a Lambertian surface is modeled. (b) Projection in the case of a $1.5 \mathrm{eV}$ top cell bandgap.

As shown on Figure 5, the operating current density of the tandem GaAsP/Si dual junction cell is subsequently very dependent of the TDD for lower than optimal top cell bandgaps. This is particularly the case for TDD values between $10^{4} \mathrm{~cm}^{-2}$ and $10^{6} \mathrm{~cm}^{-2}$, as attested by the iso-current density contours being very horizontal and close one to the other for bandgaps under $1.7 \mathrm{eV}$.

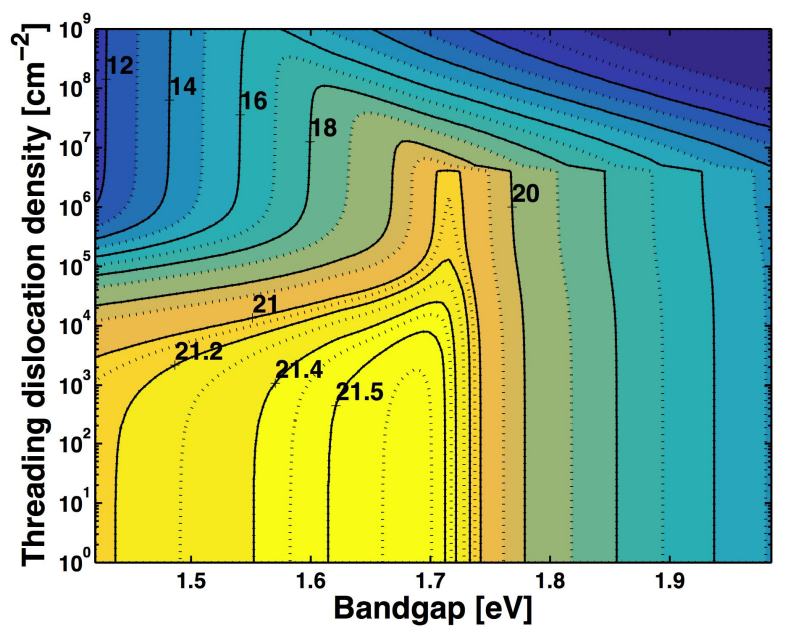

Figure 5. Operating current density of a Lambertian surface dual junction GaAsP/Si tandem solar cell as a function of the top cell bandgap and TDD. The top cell thickness is fixed at $2 \mu \mathrm{m}$. 
This is also apparent on Figure 6a, which shows the efficiency of the dual junction GaAsP/Si solar cell as a function of the TDD and the top cell bandgap, with the iso-efficiency contours being very close to one another and relatively horizontal for TDD values between $10^{4} \mathrm{~cm}^{-2}$ and $10^{6} \mathrm{~cm}^{-2}$ and top cell bandgaps under $1.7 \mathrm{eV}$. Figure $6 \mathrm{~b}$ shows the particular case of a $1.5 \mathrm{eV}$ bandgap top cell versus the bandgap-optimized case represented by the dashed line in Figure 6a. The efficiency of the dual junction with a $1.5 \mathrm{eV}$ top cell drops way faster than with a bandgap-optimized top cell for TDD values between $10^{4} \mathrm{~cm}^{-2}$ and $10^{6} \mathrm{~cm}^{-2}$, as the impact of the TDD on the performances of the top cell ads up with the quenching of the LC. Above $10^{6} \mathrm{~cm}^{-2}$ the difference in efficiency is reduced as the optimal bandgap of the top cell decreases.
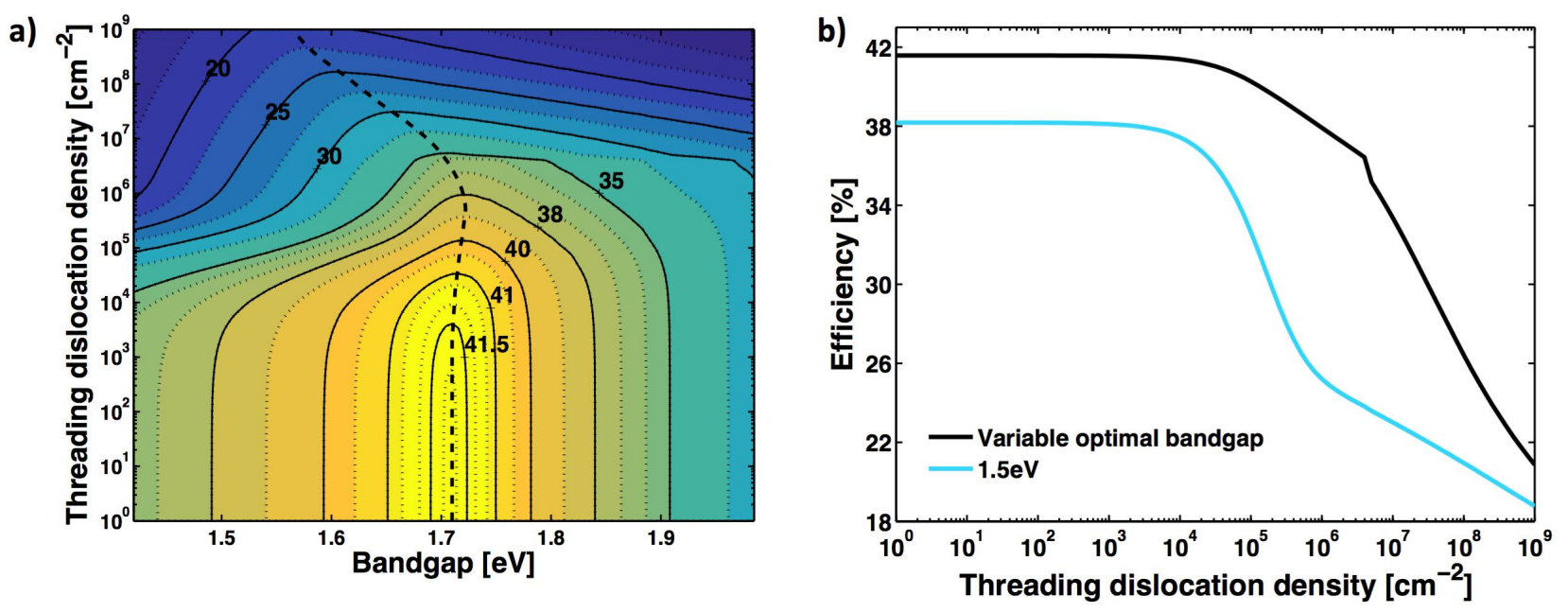

Figure 6. (a) Iso-efficiency contours of a Lambertian surface dual junction GaAsP/Si tandem solar cell as a function of the top cell bandgap and TDD. The dashed line represents the optimal bandgap as a function of the TDD. (b) Projection in the case of the optimal bandgap (black) versus $1.5 \mathrm{eV}$ (light blue) for the top cell.

As a consequence the welcome flexibility in the design and operations of the cell thanks to LC, as demonstrated in Ref. [10], is lost for TDD values over $10^{4}-10^{5} \mathrm{~cm}^{-2}$. For TDD values over $10^{6} \mathrm{~cm}^{-2}$, non-ideal conditions - such as high temperatures or an incident spectrum different from AM1.5 - will therefore have a strong negative impact on the performances of the cell. The luminescent efficiency, already known as an essential figure of merit for standard 1junction III-V cells [17], is even more critical for GaAsP/Si dual junction solar cells.

\section{CONCLUSION}

An inclusive yet simple model has been developed to investigate the impact of the threading dislocation density (TDD) and the luminescent coupling (LC) on the performances of $\mathrm{GaAsP} / \mathrm{Si}$ tandem dual junction solar cells. The model takes into account Shockley-Read-Hall (SRH) recombinations due to threading dislocations (TDs) in the GaAsP top cell, Auger recombination in the Si bottom cell, two absorption models depending on the texturing of the top surface (flat versus Lambertian) and LC between the cells due to reemitted photons from the top cell cascading to the bottom cell where they contribute to the photocurrent.

We show that, for tandem cells with a lower than optimal top cell bandgap and in the absence of TDs, LC between the top and bottom cells provides a substantial boost to the bottom cell current density, considerably reducing the impact of the non-optimized top cell bandgap-thickness combinations. This LC current density boost in the bottom cell amounts to nearly $7 \mathrm{~mA} . \mathrm{cm}^{-2}$ for a $2 \mu \mathrm{m}$-thick $1.5 \mathrm{eV}$ GaAsP top cell.

However, when TDs are taken into account, non-radiative SRH recombinations in the top cell take over radiative recombinations and the LC process is quenched. As a result, the boost to the bottom cell current density is reduced for TDDs above $10^{4} \mathrm{~cm}^{-2}$, becoming negligible over $10^{6} \mathrm{~cm}^{-2}$. This strongly impacts the tandem GaAsP/Si operating current density and its efficiency for non-optimized top cells with a too low bandgap. Consequently the flexibility in the design and operations of tandem solar cells due to LC is lost for TDD values over $10^{4}-10^{5} \mathrm{~cm}^{-2}$. Luminescent efficiency in the GaAsP top cell is therefore critical for non-optimized GaAsP/Si cells and medium to high TDD cells (TDD $>10^{4} \mathrm{~cm}^{-2}$ ) need to be tightly current-matched for high performances. 


\section{ACKNOWLEDGEMENTS}

This work is supported by Total SA. H. Liu would like to thank The Royal Society for funding his University Research Fellowship.

\section{REFERENCES}

[1] Masuko, K., Shigematsu, M., Hashiguchi, T., Fujishima, D., Kai, M., Yoshimura, N., Yamaguchi, T., Ichihashi, Y., Mishima, T., Matsubara, N., Yamanishi, T., Takahama, T., Taguchi, M., Maruyama, E. and Okamoto, S., "Achievement of More Than 25\% Conversion Efficiency With Crystalline Silicon Heterojunction Solar Cell," IEEE J. Photovolt. 4(6), 1433-1435 (2014).

[2] Smith, D. D., Cousins, P., Westerberg, S., De Jesus-Tabajonga, R., Aniero, G. and Shen, Y. C., "Toward the Practical Limits of Silicon Solar Cells," IEEE J. Photovolt. 4(6), 1465-1469 (2014).

[3] Shockley, W. and Queisser H. J., "Detailed Balance Limit of Efficiency of p-n Junction Solar Cells," J. Appl. Phys. 32(3), 510-519 (1961).

[4] Tiedje, T., Yablonovitch., E., Cody, G. D. and Brooks, B. G., "Limiting efficiency of Silicon Solar Cells," IEEE Trans. Electron Devices 34(6), 711-716 (1984).

[5] Takamoto, T., Washio, H. and Juso, H., "Application of $\mathrm{InGaP} / \mathrm{GaAs} / \mathrm{InGaAs}$ Triple Junction Solar cells to Space Use and Concentrator Photovoltaic," Proc. $40^{\text {th }}$ IEEE PVSC, 1-5 (2014).

[6] Chiu, P. T., Law, D. C., Woo, R. L., Singer, S. B., Bhusari, D., Hong, W. D., Zakaria, A., Boisvert, J., Mesropian, S., King, R. R. and Karam, N. H., " $35.8 \%$ space and $38.8 \%$ terrestrial $5 \mathrm{~J}$ direct bonded cells," Proc. $40^{\text {th }}$ IEEE PVSC, 11-13 (2014).

[7] Grassman, T. J., Brenner, M. R., Carlin, A. M., Rajagopalan, S., Unocic, R., Dehoff, R., Mills, M., Fraser, H. and Ringel, S. A., "Toward Metamorphic Multijunction GaAsP/Si Photovoltaics Grown on Optimized GaP/Si Virtual Substrates Using Anion-Graded GaAs $\mathrm{P}_{1-\mathrm{y}}$ Buffers," Proc. 34 $4^{\text {th }}$ IEEE PVSC, 2016-2021 (2009).

[8] Grassman, T. J., Brenner, M. R., Gonzalez, M, Carlin, A. M., Unocic, R., Dehoff, R., Mills, M. and Ringel S. A., "Characterization of Metamorphic GaAsP/Si Materials and Devices for Photovoltaic Applications," IEEE Trans. Electron Devices 57(12), 3361-3369 (2010).

[9] Lang, J. R., Faucher, F., Tomasulo, S., Nay Yaung, K. and Lee, M. L., "Comparison of GaAsP solar cells on GaP and GaP/Si," Appl. Phys. Lett. 103, 092102 (2013).

[10] Brown, A. S. and Green M. A., "Radiative coupling as a means to reduce spectral mismatch in monolithic tandem solar cells tacks - Theoretical considerations," Proc. $29^{\text {th }}$ IEEE PVSC, 868-871 (2002).

[11] Yablonovitch, E. and Cody, G. D., "Intensity Enhancement in Textured Optical Sheets for Solar Cells," IEEE Trans. Electron Devices 29(2), 300-305 (1982).

[12] “Reference Solar Spectral Irradiance: Air Mass 1.5," RReDC - NREL, http://rredc.nrel.gov/solar/spectra/am1.5/ (23 September 2014).

[13] Würfel, P., “The Chemical Potential of Radiation,” J. Phys. C Solid State Phys. 15(18), 3967-3985 (1982).

[14] Sinton, R. A. and Swanson, R. M., "Recombination in Highly Injected Silicon," IEEE Trans. Electron Devices 34(6), 1380-1389 (1987).

[15] Yamaguchi, M. and Amano, C., "Efficiency calculations of thin-film GaAs solar cells on Si substrates," J. Appl. Phys. 58(9), 3601-3606 (1985).

[16] "Basic parameters, Band structure and carrier concentration and Electrical Parameters of $\mathrm{Ga}_{\mathrm{x}} \mathrm{In}_{1-\mathrm{x}} \mathrm{As}_{\mathrm{y}} \mathrm{P}_{1-\mathrm{y}}$," Ioffe Physical Technical Institute, http://www.ioffe.ru/SVA/NSM/Semicond/GaInAsP/index.html (23 September 2014).

[17] Miller, O. D., Yablonovitch, E. and Kurtz, S.R., "Strong Internal and External Luminescence as Solar Cells Approach the Shockley-Queisser Limit," IEEE J. Photovolt. 2(3), 303-311 (2012). 